\title{
Metabolic pathway engineering for production of 1,2-propanediol and 1-propanol by Corynebacterium glutamicum
}

\author{
Daniel Siebert and Volker F. Wendisch ${ }^{*}$
}

\begin{abstract}
Background: Production of the versatile bulk chemical 1,2-propanediol and the potential biofuel 1-propanol is still dependent on petroleum, but some approaches to establish bio-based production from renewable feed stocks and to avoid toxic intermediates have been described. The biotechnological workhorse Corynebacterium glutamicum has also been shown to be able to overproduce 1,2-propanediol by metabolic engineering. Additionally, C. glutamicum has previously been engineered for production of the biofuels ethanol and isobutanol but not for 1-propanol.

Results: In this study, the improved production of 1,2-propanediol by C. glutamicum is presented. The product yield of a C. glutamicum strain expressing the heterologous genes gldA and mgsA from Escherichia coli that encode methylglyoxal synthase gene and glycerol dehydrogenase, respectively, was improved by additional expression of alcohol dehydrogenase gene yqhD from E. coli leading to a yield of $0.131 \mathrm{~mol} / \mathrm{mol}$ glucose. Deletion of the endogenous genes $h d p A$ and Idh encoding dihydroxyacetone phosphate phosphatase and lactate dehydrogenase, respectively, prevented formation of glycerol and lactate as by-products and improved the yield to $0.343 \mathrm{~mol} / \mathrm{mol}$ glucose. To construct a 1-propanol producer, the operon ppdABC from Klebsiella oxytoca encoding diol dehydratase was expressed in the improved 1,2-propanediol producing strain ending up with $12 \mathrm{mM}$ 1-propanol and up to $60 \mathrm{mM}$ unconverted 1,2-propanediol. Thus, $\mathrm{B}_{12}$-dependent diol dehydratase activity may be limiting 1-propanol production.

Conclusions: Production of 1,2-propanediol by C. glutamicum was improved by metabolic engineering targeting endogenous enzymes. Furthermore, to the best of our knowledge, production of 1-propanol by recombinant C. glutamicum was demonstrated for the first time.
\end{abstract}

Keywords: Corynebacterium glutamicum, Metabolic engineering, 1-propanol, 1,2-propanediol

\section{Background}

The usage of 1,2-propanediol ranges from building blocks in plastics industry, in de-icing and anti-freeze fluids, and as additive in cosmetics, nutrition, medicines, dyes, and liquid detergents [1]. Due to the very broad spectrum of applications of the bulk chemical 1,2-propanediol, also known as propylene glycol, annually over 1 billion pounds of 1,2-propanediol are sold in the United States and at least 1.2 million tons are consumed worldwide [2]. To date, most of this demand is accommodated by petrochemistry. In the main route, the steam cracking product propylene [3] is converted to propylene oxide

\footnotetext{
* Correspondence: volker.wendisch@uni-bielefeld.de

Chair of Genetics of Prokaryotes, Faculty of Biology and CeBiTec, Bielefeld University, Universitätsstr. 25, 33615 Bielefeld, Germany
}

$[4,5]$, which is further hydrolyzed to 1,2-propanediol [6]. The occurrence of toxic intermediates and sideproducts initiated efforts to find more sustainable and less toxic routes, e.g., by fermentation of renewable carbon sources by microorganisms. Various microorganisms showing potential to produce 1,2-propanediol from renewable feed stocks have been described, e.g., Clostridium thermosaccharolyticum [7], Saccharomyces cerevisiae [8, 9], Escherichia coli [1, 10], Synechoccus elongates [11], and Corynebacterium glutamicum [12].

The Gram-positive and generally-recognized-as-safe rod-shaped soil bacterium Corynebacterium glutamicum [13] is the main source of the worldwide production of the amino acids glutamate and lysine in a scale of over 5 million tons per year [14]. A wealth of information on C. glutamicum exists [14-18] including sequencing 
its genome [19] and creating a genome-streamlined chassis organism [20]. Metabolic engineering aimed at the production of not only many other amino acids $[14,21]$ but also for example at monomers of bioplastics (e.g., cadaverine [22, 23] and putrescine [23]), organic acids [24], carotenoids [25], and biofuels. C. glutamicum was engineered for isobutanol production and shown to exhibit less toxicity to isobutanol than E. coli $[26,27]$. The isobutanol yield by recombinant C. glutamicum was competitive with E. coli [28]. In particular, overproduction of the biofuel ethanol under oxygen deprivation conditions is well-described for C. glutamicum and shown to be efficient [29-31]. Importantly, under these conditions C. glutamicum showed high tolerance to organic acid, furan, and phenolic inhibitors present in lignocellulose hydrolysates [30]. Thus, C. glutamicum is a promising alternative biofuel production host. To enable sustainable production from several alternative carbon sources, the substrate spectrum of C. glutamicum was widened by metabolic engineering [32]. Since 1,2-propanediol production by $C$. glutamicum has been shown [12] in principle, this study aimed at improving 1,2-propanediol production and at producing 1-propanol as derived compound. This primary alcohol, also named npropanol, finds application in the solvent, cosmetic, and pharmaceutical industries, in antiseptic solutions, as precursor for diesel fuels and in the plastics industry and finally as biofuel [33-35]. C. glutamicum has previously been engineered for production of the biofuels ethanol [31] and isobutanol [26-28] but not for 1-propanol. Natural microorganisms are not known to secrete significant amounts of 1-propanol. However, Propionibacterium freudenreichii has been engineered for the direct conversion of propionyl-CoA to 1-propanol [34]. Engineered E. coli strains either convert 2-ketobutyrate to 1-propanol by variants of the threonine and citramalate pathways [36, 37] or by extending succinate dissimilation [35]. Finally, 1,2-propanediol can be converted in a two-step conversion to 1-propanol by diol dehydratase from Klebsiella oxytoca [33]. The latter pathway was chosen in this study for construction of a C. glutamicum 1-propanol-producing strain.

\section{Results}

\section{Co-overexpression of yqhD from E. coli increased 1,2-propanediol production}

C. glutamicum has previously been engineered for 1,2propanediol production by expressing the heterologous genes $m g s A$ and gldA encoding methylglyoxal synthase gene and glycerol dehydrogenase from E. coli [12]. Expression of these genes as artificial operon from the plasmid pEKEx3-mgsA-gldA in C. glutamicum WT yielded $19 \pm$ $1 \mathrm{mM}$ 1,2-propanediol within $51 \mathrm{~h}$ (Fig. 2) when using modified CGXII minimal medium with a decreased nitrogen content $(5 \mathrm{~g} / \mathrm{L}$ ammonium sulfate) and $184 \pm 1 \mathrm{mM}$ glucose as sole carbon source. Thus, the base strain produced 1,2-propanediol with a yield of $0.103 \mathrm{~mol} / \mathrm{mol}$ glucose.

Methylglyoxal is a toxic intermediate of the conversion of dihydroxyacetone phosphate (DHAP) to 1,2-propanediol (Fig. 1), and in E. coli, additional overexpression of the alcohol dehydrogenase genes $y q h D$ or fucO was shown to increase the yield of 1,2-propanediol from glycerol [10]. Heterologous expression of $y q h D$ with $m g s A$ and gldA from plasmid pEKEx3-mgsA-yqhD-gldA in C. glutamicum WT improved 1,2-propanediol production by about $27 \%$ as $24 \pm 1 \mathrm{mM} \mathrm{1,2-propanediol} \mathrm{accumulated} \mathrm{after} 51 \mathrm{~h}$ (Fig. 2b), which correlated to a product yield of $0.131 \mathrm{~mol} /$ mol. Both C. glutamicum WT(pEKEx3-mgsA-gldA) and WT(pEKEx3-mgsA-yqhD-gldA) grew and utilized glucose as growth substrate slightly slower than the empty vector carrying control strain C. glutamicum WT(pEKEx3) (Fig. 2a). The addition of alcohol dehydrogenase gene fucO as fourth gene of the heterologously expressed operon on plasmid pEKEx3-mgsA-yqhD-fucO-gldA did not further improve 1,2-propanediol production as compared to WT(pEKEx3-mgsA-yqhD-gldA) (data not shown).

A comparison between strains WT(pEKEx3-mgsA-gldA) and WT(pEKEx3-mgsA-yqhD-gldA) with respect to byproduct formation revealed that acetol, the direct precursor of 1,2-propanediol (Fig. 1), accumulated to higher concentrations in supernatants of WT(pEKEx3-mgsA-gldA) than of WT(pEKEx3-mgsA-yqhD-gldA), i.e., $14 \mathrm{mM}$ as compared $5 \mathrm{mM}$, after glucose was depleted (Fig. $2 \mathrm{~b}$ ). On the other hand, WT(pEKEx3-mgsA-gldA) only produced $8 \pm$ $1 \mathrm{mM}$ glycerol as a by-product, whereas the additional expression of $y q h D$ resulted in accumulation of $42 \pm 1 \mathrm{mM}$ (Fig. 2c). Interestingly, the empty vector control produced $32 \pm 3 \mathrm{mM}$ dihydroxyacetone (DHA), while C. glutamicum strains WT(pEKEx3-mgsA-gldA) and WT(pEKEx3$m g s A-y q h D$-gldA) accumulated less than $5 \mathrm{mM}$ DHA (Fig. 2c). Thus, preventing glycerol formation by the so far best producing strain WT(pEKEx3-mgsA-yqhDgldA) offers the potential to improve 1,2-propanediol production.

\section{Stopping glycerol formation by deleting the gene hdpA resulted in higher yields of 1,2-propanediol}

Typically, glycerol is hardly secreted by C. glutamicum WT, although two enzymes involved in glycerol formation have been found, namely gpp-encoded glycerol-3-phosphatase [38] and butA-encoded (S,S)-butanediol dehydrogenase [39]. In the experiments described above, glycerol was produced by the recombinant strains WT(pEKEx3$m g s A$-gldA) and WT(pEKEx3-mgsA-yqhD-gldA) but nearly not by the parent strain WT(pEKEx3). This indicated that the heterologous enzymes present in these recombinants may be involved in glycerol formation. Since it is known that the gldA-encoded glycerol 


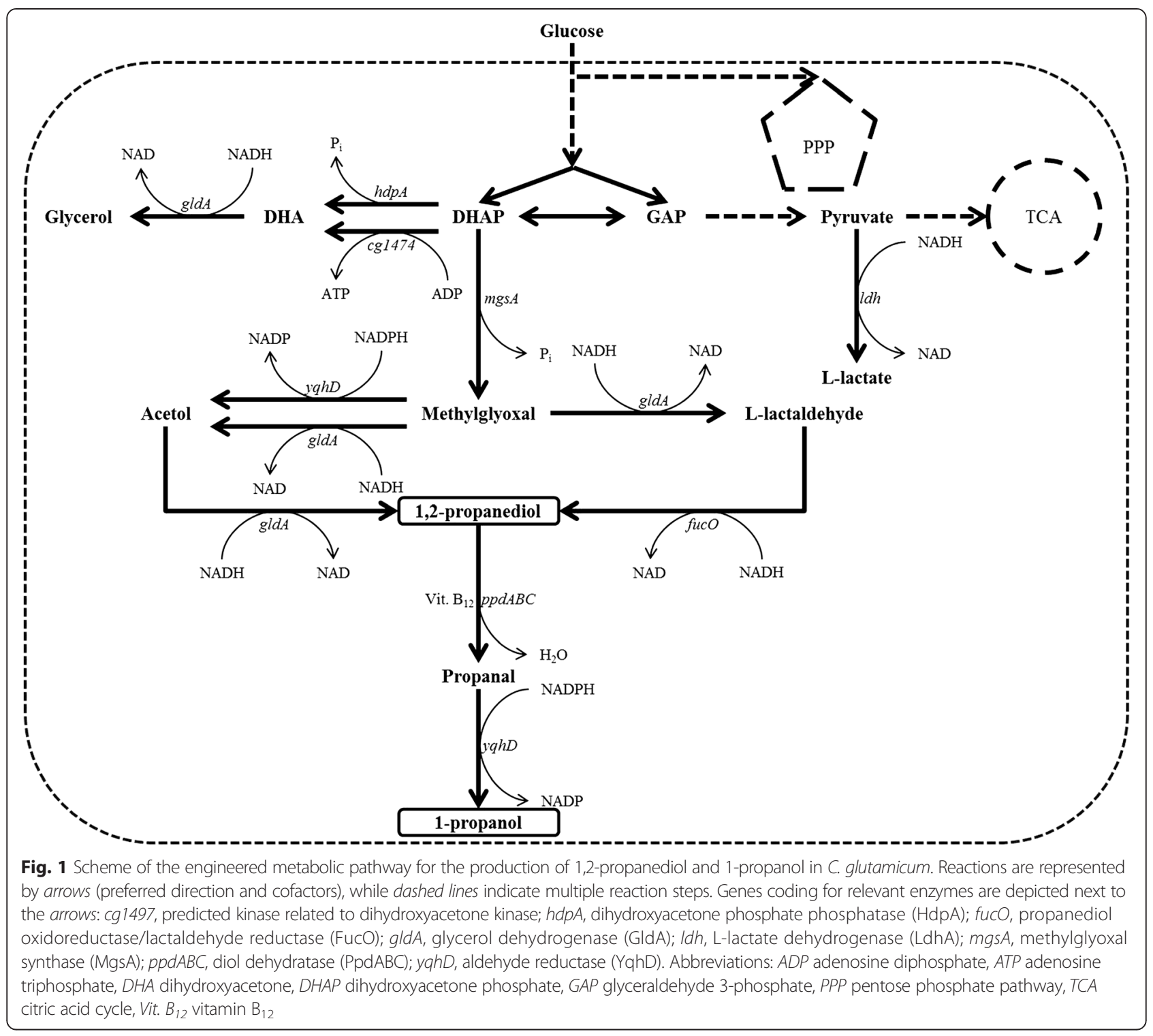

dehydratase from $E$. coli accepts also dihydroxyacetone, acetol, and methylglyoxal as substrates [40] (Fig. 1), it was tested if dihydroxyacetone formation can be prevented. Secretion of dihydroxyacetone by C. glutamicum WT occurs under certain conditions, e.g., acidic conditions [41], and was observed for WT(pEKEx3) under the conditions of 1,2-propanediol production described above. Two enzymes may be involved in DHA production, namely DHAP phosphatase encoded by $h d p A$ [42] and a predicted kinase related to dihydroxyacetone kinases encoded by cg1497 [43]. To test if these enzymes are relevant for glycerol formation from DHA by the 1,2-propanediol-producing strain WT(pEKEx3$m g s A-y q h D-g l d A$ ), both genes were deleted by homologous recombination individually and in combination. The resulting strains C. glutamicum $\Delta$ cg1497(pEKEx3- $m g s A-y q h D$-gldA $), \Delta h d p A(\mathrm{pEKEx} 3-m g s A-y q h D$-gldA), and $\Delta \operatorname{cg} 1497 \Delta h d p A(\mathrm{pEKEx} 3-m g s A-y q h D$-gldA) were grown as described above for WT(pEKEx3-mgsA-yqhD-gldA). The deletion of the gene cg1497 had no impact on the 1,2-propanediol formation (data not shown). Upon deletion of $h d p A$, 1,2-propanediol production increased by about $90 \%$ (Fig. 3b), while the double deletion mutant showed no further increase (data not shown). After $51 \mathrm{~h}$ of cultivation, C. glutamicum $\Delta h d p A(\mathrm{pEKEx} 3-m g s A-y q h D-g l d A)$ accumulated $46 \pm 4 \mathrm{mM}$ 1,2-propanediol, which corresponds to a product yield of $0.249 \mathrm{~mol} / \mathrm{mol}$. C. glutamicum WT (pEKEx3-mgsA-yqhD-gldA) and $\Delta h d p A$ (pEKEx3-mgsA$y q h D$-gldA) grew with comparable growth rates, utilized glucose comparably fast (Fig. 3a), and accumulated comparable concentrations ( 5 and $7 \mathrm{mM}$, respectively). However, glycerol was not a significant by-product $(<5 \mathrm{mM})$ of 


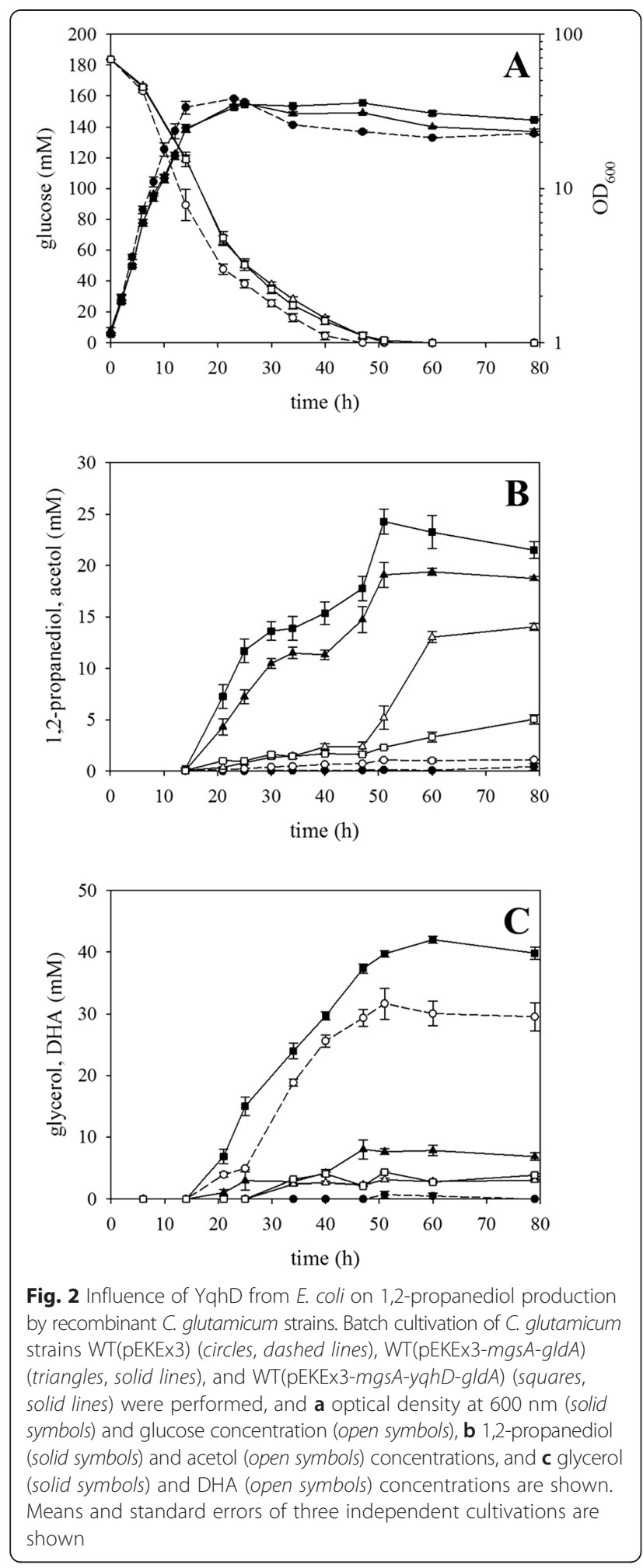

the $h d p A$ deletion strain, while the parental strain accumulated more than $40 \mathrm{mM}$ glycerol (Fig. 3c). Thus, preventing DHA formation from DHAP by deletion of $h d p A$ prevented subsequent formation of glycerol from DHA and improved 1,2-propanediol production.
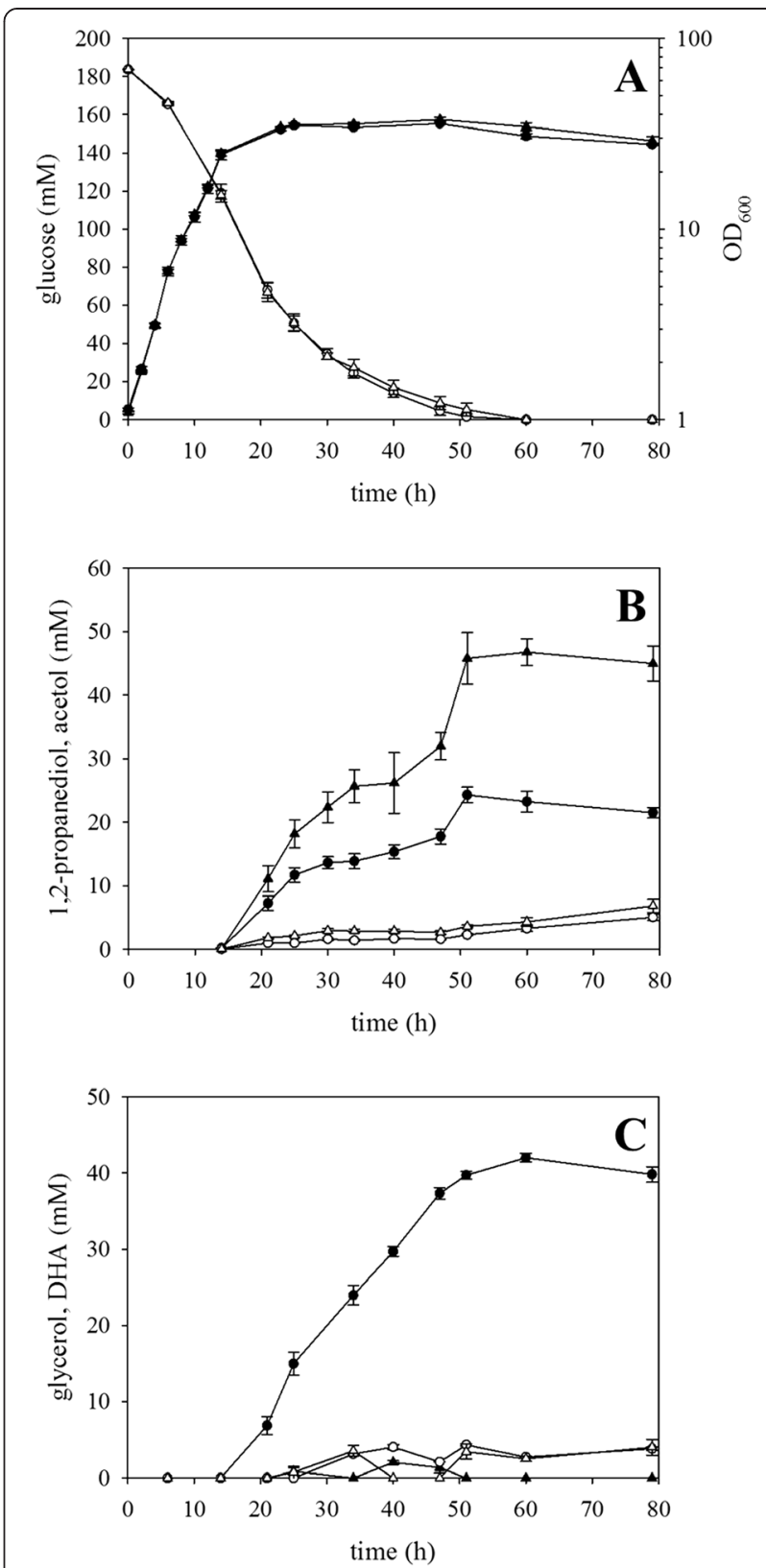

Fig. 3 Influence of endogenous DHAP phosphatase HdpA on 1,2-propanediol production by recombinant C. glutamicum strains. Batch cultivation of C. glutamicum WT(pEKEx3-mgsA-yqhD-gldA) (circles) and $\Delta h d p A(p E K E x 3-m g s A-y q h D-g l d A)$ (triangles) were performed, and a optical density at $600 \mathrm{~nm}$ (solid symbols) and glucose concentration (open symbo/s), b 1,2-propanediol (solid symbo/s) and acetol (open symbols) concentrations, and c glycerol (solid symbols) and DHA (open symbols) concentrations are shown. Means and standard errors of three independent cultivations are shown

\section{Deleting Idh prevented transient L-lactate accumulation} and led to faster and higher 1,2-propanediol production The deletion of $h d p A$ prevented formation of about $40 \mathrm{mM}$ glycerol but increased 1,2-propanediol accumulation by about $22 \mathrm{mM}$ only (Fig. 3). Since 1,2-propanediol 
is more reduced than glycerol and since it is known that C. glutamicum utilizes excess NADH to reduce pyruvate to L-lactate, lactate formation may compete with 1,2-propanediol formation for NADH. In C. glutamicum, L-lactate is formed by fermentative, NADH-dependent lactate dehydrogenase LdhA under oxygen deprivation conditions [44] but transiently also during aerobic cultivation [45]. Re-uptake and re-utilization of lactate does not generate NADH but menaquinol, because both L- and D-lactate dehydrogenases LldD and Dld oxidize lactate to pyruvate in menaquinone-dependent reactions $[45,46]$. Thus, $l d h$ was deleted and the resulting strain C. glutamicum $\Delta h d p A \Delta l d h(\mathrm{pEKEx} 3-m g s A-y q h D-g l d A)$ was compared to strain $\triangle h d p A$ (pEKEx3-mgsA-yqhD-gldA) in batch cultivations. As consequence of introducing the $l d h$ deletion, 1,2-propanediol production increased by about $38 \%$. C. glutamicum strain $\Delta h d p A \Delta l d h(\mathrm{pEKEx} 3-$ mgsA-yqhD-gldA) accumulated $63 \pm 4 \mathrm{mM} \mathrm{1,2-propane-}$ diol (Fig. 4b), which corresponds to a product yield of $0.343 \mathrm{~mol} / \mathrm{mol}$. Moreover, the $l d h$ deletion strain utilized glucose faster and accumulated 1,2-propanediol faster than the parental strain, while the growth rates of both strains were comparable (Fig. 4a). Neither DHA nor glycerol accumulated to significant concentrations $(<5 \mathrm{mM})$, but more acetol (15 mM as compared to $7 \mathrm{mM}$ ) was produced by the $l d h$ deletion strain (Fig. 4b). Lactate formation by the $l d h$ deletion strain was not detectable $(<1 \mathrm{mM})$, while the parental strains and all other strains mentioned in Figs. 2, 3, and 4 accumulated lactate to low concentrations (between 1 and $4 \mathrm{mM}$ ) over the whole fermentation process. Taken together, $l d h$ deletion improved 1,2-propanediol production considerably.

\section{Production of 1-propanol by recombinant C. glutamicum}

A 1,2-propanediol-producing $E$. coli strain produced 1propanol when the ppdABC operon from $K$. oxytoca, which encodes a vitamin $B_{12}$-dependent 1,2-propanediol dehydratase, was expressed [33, 47]. After vitamin $B_{12^{-}}$ dependent 1,2-propanediol dehydratase has converted 1,2-propanediol to 1-propanal, the latter is reduced to 1propanol by alcohol dehydrogenases such as YqhD [48]. Thus, the operon ppdABC of $K$. oxytoca was cloned into the expression vector pVWEx1, which is compatible with expression vector pEKEx3, and used to transform 1,2propanediol-producing strains. Cultivated in minimal medium with $217 \pm 1 \mathrm{mM}$ glucose and $10 \mu \mathrm{M}$ vitamin $\mathrm{B}_{12}$, C. glutamicum strain $\Delta h d p A \Delta l d h$ (pEKEx3-mgsA$y q h D$-gldA)(pVWEx1-ppdABC) accumulated 1-propanol to the highest concentration $(12 \pm 1 \mathrm{mM})$ after $70 \mathrm{~h}$ (Fig. 5a). This strain did not accumulate significant concentrations of glycerol, DHA, and acetol (data not shown). However, 1,2-propanediol was still the main product $(62 \pm 2 \mathrm{mM})$.
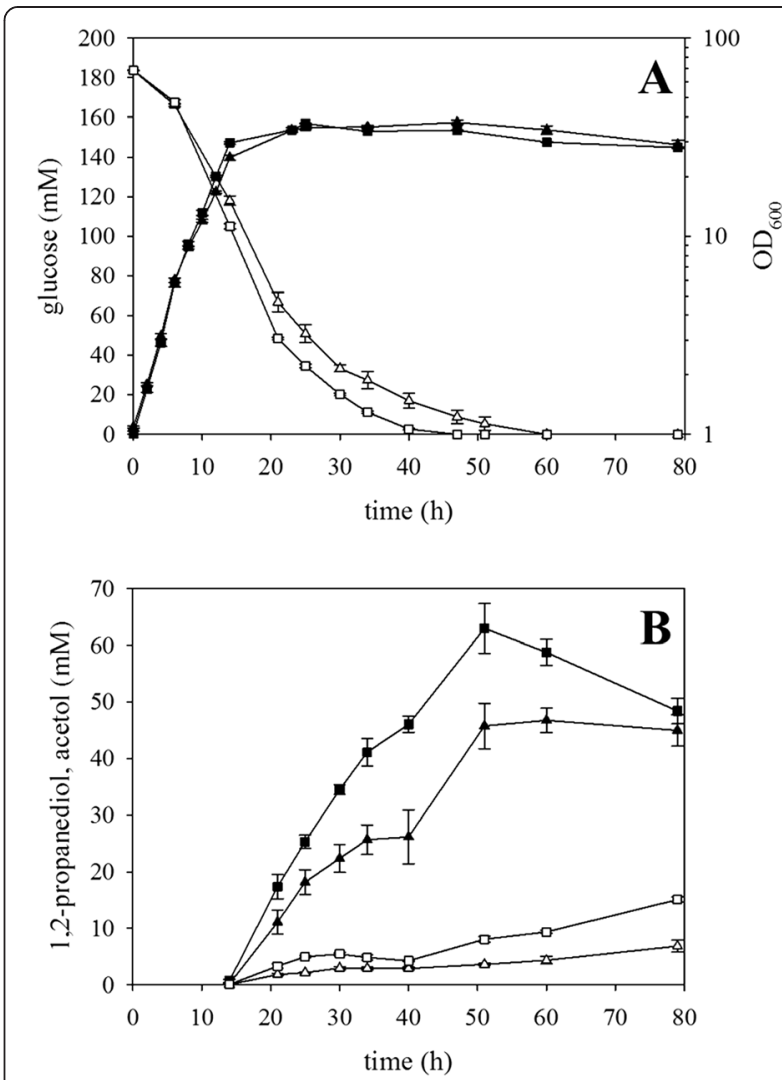

Fig. 4 Influence of endogenous NADH-dependent L-lactate dehydrogenase $L d h$ on 1,2-propanediol production by recombinant $C$. glutamicum strains. Batch cultivations of $C$. glutamicum $\triangle h d p A($ pEKEX3mgsA-yqhD-gldA) (triangles) and $\Delta h d p A \Delta / d h$ (pEKEx3-mgsA-yqhD-gldA) (squares) were performed, and a optical density at $600 \mathrm{~nm}$ (solid symbols) and glucose concentration (open symbols) and b 1,2propanediol (solid symbols) and acetol (open symbols) concentrations are shown. Means and standard errors of three independent cultivations are shown

As expected from the 1,2-propanediol production experiments, deletions of genes $h d p A$ and $l d h$ were beneficial for 1-propanol production since strain WT(pEKEx3-mgsA$y q h D-g l d A)(\mathrm{pVWEx} 1-p p d A B C)$ accumulated almost twofold less 1-propanol (7 $\pm 1 \mathrm{mM})$ and 1,2-propanediol (30 \pm $1 \mathrm{mM}$; Fig. 5b).

Strain WT(pEKEx3-mgsA-gldA)(pVWEx1-ppdABC) that did not overexpress $y q h D$ from $E$. coli, which presumably is involved in reduction of 1-propanal to 1-propanol, only accumulated $2 \pm 1 \mathrm{mM}$ 1-propanol and utilized glucose incompletely (Fig. 5a). Accordingly, this strain only produced $9 \pm 2 \mathrm{mM}$ 1,2-propanediol and $43 \pm 4 \mathrm{mM}$ glycerol (Fig. 5c).

Taken together, 1-propanol was produced for the first time by recombinant $C$. glutamicum and strain $\Delta h d p A \Delta l d h$ (pEKEx3-mgsA-yqhD-gldA)(pVWEx1-ppdABC) accumulated 1-propanol up to a concentration of $12 \mathrm{mM}$. Besides vitamin $\mathrm{B}_{12}$-dependent 1,2-propanediol dehydratase, also 

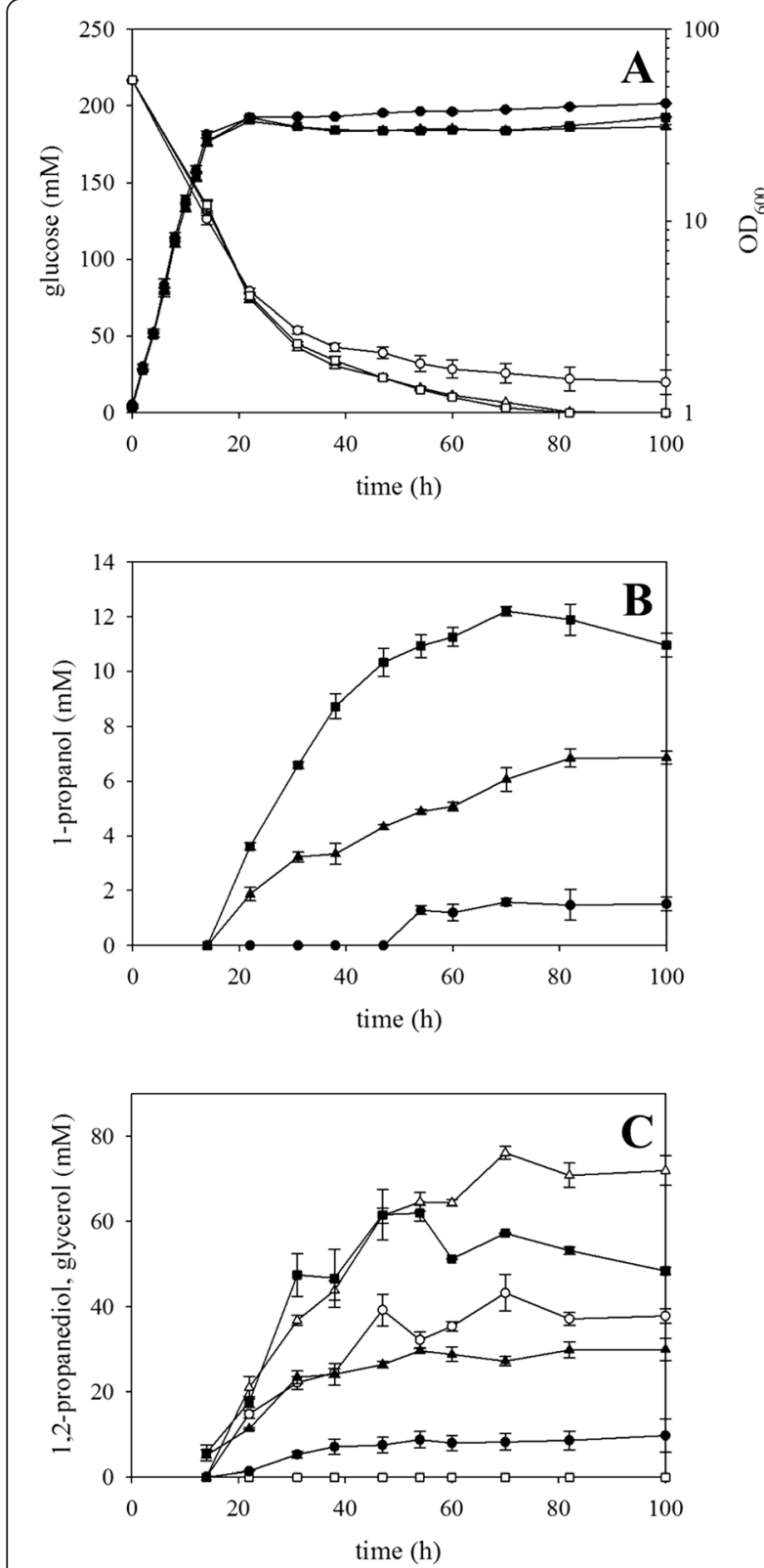

Fig. 5 Production of 1-propanol by recombinant C. glutamicum strains. Batch cultivation of C. glutamicum WT(pEKEx3-mgsA-gldA)(pVWEx1ppdABC) (circles), WT(pEKEx3-mgsA-yqhD-gldA)(pWWEx1-ppdABC) (triangles), and $\Delta h d p A \Delta / d h(p E K E \times 3-m g s A-y q h D-g l d A)(p W W E x 1-p p d A B C)$ (squares) were performed, and a optical density at $600 \mathrm{~nm}$ (solid symbo/s) and glucose concentration (open symbo/s), b 1-propanol concentrations, and c 1,2-propanediol (solid symbols) and glycerol (open symbo/s) concentrations are shown. Means and standard errors of three independent cultivations are shown

alcohol dehydrogenase YqhD appeared to be involved in converting 1,2-propanediol to 1-propanol.

\section{Discussion}

In this study, production of 1,2-propanediol by C. glutamicum was improved and production of the biofuel molecule 1-propanol by C. glutamicum was shown for the first time. It has been shown previously that expression of the heterologous methylglyoxal synthase gene $m g s A$ from $E$. coli was required for 1,2-propanediol and had to be coupled with glycerol dehydrogenase either encoded by heterologous gene gldA from E. coli or endogenous cgR_2242 [12]. Within $96 \mathrm{~h}$, up to $25 \mathrm{mM}$ 1,2-propanediol and $44 \mathrm{mM}$ acetol were produced from $333 \mathrm{mM}$ glucose as a sole carbon source [12]. Using a comparable strain but the cultivation setup employed in this study, it was possible to produce $19 \mathrm{mM} \mathrm{1,2-propanediol} \mathrm{in} 51 \mathrm{~h}$ from $184 \mathrm{mM}$ glucose by overexpression of $m g s A$ and $g l d A$ from E. coli in C. glutamicum WT (Fig. 2). Notably, accumulation of 1,2-propanediol and side products started after the cells entered the stationary phase, thus, production was not coupled to growth (Fig. 2).

Alcohol dehydrogenase YqhD proved beneficial for 1,2-propanediol production (increased by $27 \%$ to a yield of $0.131 \mathrm{~mol} / \mathrm{mol}$ glucose, Fig. 2), presumably because conversion of methylglyoxal to acetol and 1,2-propanediol was improved by YqhD. This enzyme has the following characteristics: a reductase activity for at least 12 aldehydes and thus increasing tolerance to aldehydes as aldehyde scavenger; preferring aldehydes over alcohols as substrates; a better conversion of alcohols longer than three carbon atoms; dependence of NADPH/NADP and divalent cations (e.g., zinc) as cofactors [48]. Notably, YqhD is NADPHdependent [48] as compared to the NADH-dependent GldA, thus, YqhD is coupled to anabolic metabolism, which is driven by NADPH. Overexpression of $y q h D$ proved beneficial for production of, e.g., 3-hydroxypropionic acid by $E$. coli [49], poly(3-hydroxypropionate) from glycerol using engineered Klebsiella pneumoniae [50], short-chain alcohols by E. coli [51], or acetol by E. coli [52].

Heterologous expression of gldA and $y q h D$ from E. coli resulted in production of the side-product glycerol since these aldehyde reductases reduced DHA to glycerol [40]. Two possible enzymes were considered to be involved in the reduction of DHA metabolism, namely cg1497 and $h d p A[42,43]$. Only the deletion of $h d p A$ prevented glycerol formation and improved 1,2-propanediol production increasing the yield by about $90 \%$ up to $0.249 \mathrm{~mol} / \mathrm{mol}$ glucose (Fig. 3). The strain lacking endogenous $h d p A$ showed improved 1,2-propanediol production due to two possible advantages. First of all, DHAP is not converted to DHA and, thus, supply of DHAP for the MgsA reaction to methylglyoxal was improved. Secondly, preventing reduction of DHA to glycerol increased provision of the redox cofactor NADH for the reactions converting methylglyoxal to 1,2-propanediol. Formation of glycerol as sideproduct of $C$. glutamicum strains expressing heterologous gldA and/or $y q h D$ is distinct from glycerol production of C. glutamicum WT. In C. glutamicum WT, glycerol is formed from glycerol 3-phosphate by glycerol 3-phosphate 
phosphatase Gpp [38]. Since C. glutamicum WT secretes DHA under certain condition [41, 42], it is devoid of an enzyme catalyzing reduction of DHA to glycerol as efficient as observed in recombinants expressing heterologous gldA and/or $y q h D$ from $E$. coli.

With the additional deletion of the gene $l d h$, it was possible to further increase the 1,2-propanediol production by about $38 \%$ resulting in a yield of $0.343 \mathrm{~mol} / \mathrm{mol}$ (Fig. 4). Deletion of $l d h$ is a common strategy to improve production of organic acids under oxygen deprivation conditions [53, 54] since L-lactate is secreted by $C$. glutamicum under conditions of excess NADH. Two factors may have led to improved 1,2-propanediol production as result of $l d h$ deletion. Firstly, provision of NADH for reduction of methylglyoxal to acetol and 1,2-propanediol is increased since pyruvate is not reduced to L-lactate. Secondly, pyruvate and possibly also other intermediates of glycolysis may accumulate as consequence of $l d h$ deletion. This accumulation is plausible since deletion of pyruvate kinase Pyk led to accumulation of pyruvate and other glycolytic intermediates $[55,56]$. In E. coli, methylglyoxal reacts spontaneously with glutathione to form a hemithioacetal, followed by detoxification by the glycoxalase system leading to the production of D-lactate [57]. C. glutamicum lacks glutathione but possesses mycothiol as its primary low molecular weight thiol [58]. A number of mycothiol-dependent reactions have been described for C. glutamicum including formaldehyde oxidation to formate $[59,60]$. Although the reaction between mycothiol and methylglyoxal is currently not known in C. glutamicum, the overexpression of $m s h A-$ encoding mycothiol glycosyltransferase led to an increased robustness towards methylglyoxal [61].

Provision of $\mathrm{NAD}(\mathrm{P}) \mathrm{H}$ for reduction of acetol to $1,2-$ propanediol may still be limiting since even strain $C$. glutamicum $\Delta h d p A \Delta l d h$ produced up to $15 \mathrm{mM}$ acetol (Fig. 4). Notably, the accumulation of acetol increased after glucose was depleted while the 1,2-propanediol concentration decreased. Thus, 1,2-propanediol may be taken up again and oxidized to acetol to generate NADH, which may provide the cells with ATP in oxidative phosphorylation. Currently, it is not known whether oxidation of 1,2propanediol occurs via the heterologous GldA from $E$. coli or by an endogenous enzyme. Interestingly, in a recombinant cyanobacterium producing 1,2-propanediol, alternative NADPH-alcohol dehydrogenases led to higher 1,2-propanediol titers, while acetol was not produced as side-product [11].

Additionally, the production of 1-propanol by C. glutamicum is reported for the first time in this study. Heterologous expression of the operon ppdABC from $K$. oxytoca encoding diol dehydratase in a 1,2-propanediol producing C. glutamicum strain was required for 1-propanol production of up to $12 \mathrm{mM}$ (Fig. 5). Diol dehydratase PpdABC has the following characteristics: consisting of three subunits $(\alpha, \beta$, and $\gamma)$ with two units of a heterotrimer building the quaternary structure; indicated that the $\alpha$ - and $\gamma$-subunit promote the correct folding of each subunit; substrates are 1,2-propanediol, glycerol and 1,2-ethanediol with $K m$ values of $0.08 \mu \mathrm{M}, 0.73 \mathrm{mM}$, and $0.56 \mathrm{mM}$, respectively; lack of stereospecificity accepting $(R)$ - and (S)-1,2-propanediol; dependent of adenosylcobalamin and divalent cations (e.g., potassium) as cofactors [62-64]. The observation that 1,2-propanediol was still the major product (up to $62 \mathrm{mM}$; Fig. 5) indicated that 1,2-propanediol to is not converted efficiently to 1 -propanol by $B_{12}$ dependent diol dehydratase PpdABC and YqhD. However, vitamin $B_{12}$ may be limiting since it is not known if $C$. glutamicum can synthesize vitamin $\mathrm{B}_{12}$. In addition, provision of the cofactor NADPH may be a bottleneck.

There is potential for improving 1-propanol production with C. glutamicum as exemplified for E. coli [33, 47]. Overexpression of $p p d A B C$ in E. coli BW25113 for conversion of DHAP to 1,2-propanediol yielded $0.036 \mathrm{~mol} /$ mol 1-propanol from glucose [33], which is comparable to the yield of $0.032 \mathrm{~mol} / \mathrm{mol}$ reported here (Fig. 5). The yield with $C$. glutamicum doubled as consequence of deleting $l d h$ and $h d p A$ (Fig. 5). Jain et al. (2014) optimized 1-propanol production by E. coli further [47]. The improvements included co-cultivation of one strain converting glucose to 1,2-propanediol and a second strain converting 1,2-propanediol to 1-propanol [47]. The first strain was improved by overexpressing an optimized gene set for conversion of DHAP to 1,2-propanediol and by deleting four genes to improve NADH provision [47]. Furthermore, heterologous expression of a gene coding for formate dehydrogenase and feeding the additional carbon source sodium formate and yeast extract improved the redox balance [47]. The second strain harbored a synthetic diol dehydratase gene cluster with optimized gene order $(p p d A-C-B)$ and separation by linker sequences [47]. These metabolic engineering and medium optimization approaches may be helpful for improving 1-propanol production by the C. glutamicum strains described in this study. A number of engineering strategies to improve NADPH provision in C. glutamicum have been developed and include, e.g., transmembrane transhydrogenase PntAB [65], phosphoglucose isomerase mutants [66], NADPH-dependent glyceraldehyde-3phosphate dehydrogenase [67], or NAD kinase [68]. Thus, production of 1-propanol may be increased further over the proof-of-concept established in this study.

\section{Conclusions}

Metabolic engineering improved 1,2-propanediol production by $C$. glutamicum. Deletion of the endogenous genes $h d p A$ and $l d h$ combined with overexpression of the $E$. coli genes $m g s A, g l d A$, and $y q h D$ resulted in strain producing 
1,2-propanediol from glucose in mineral salt medium with a product yield of $0.343 \mathrm{~mol} / \mathrm{mol}$. Further strain engineering led to strain capable of producing 1-propanol. This is the first report of 1-propanol production by recombinant C. glutamicum.

\section{Materials and methods Microorganisms, media, and cultivation conditions}

In Table 1, all C. glutamicum strains and plasmids which were used for this study are presented. The E. coli strain DH5 $\alpha$ [69] was used for the plasmid construction and was cultured in lysogeny broth complex medium (LB) [70]. Precultivation of C. glutamicum was performed in LB with $2 \%$ glucose by inoculation from LB plates. For the main cultures of $C$. glutamicum, the cells of an overnight preculture were harvested by centrifugation (10 min; $3220 \times \mathrm{g}$ ) and transferring the appropriate volume for an optical density $(\lambda=600 \mathrm{~nm})\left(\mathrm{OD}_{600}\right)$ of 1 in $50-\mathrm{mL}$ cultures. These cells were washed with CGXII minimal medium [71] without carbon source and without urea and ammonium sulfate. The cells were again centrifuged and resuspended with the same CGXII. As sole nitrogen source $5 \mathrm{~g} / \mathrm{L}$ ammonium sulfate were added and as sole carbon source, glucose was used in the measured concentration given in the results. All cultivations of
C. glutamicum were carried out in a volume of $50 \mathrm{~mL}$ in $500-\mathrm{mL}$ baffled flasks at $30{ }^{\circ} \mathrm{C}$ and $120 \mathrm{rpm}$. The gene expression was induced by adding $1 \mathrm{mM}$ isopropyl$\beta$-D-thiogalactopyranoside (IPTG) at inoculation of the main culture. When appropriate, the medium was supplemented with $25 \mu \mathrm{g} / \mathrm{mL}$ kanamycin and $100 \mu \mathrm{g} / \mathrm{mL}$ spectinomycin. For 1-propanol production, it was necessary to add $10 \mu \mathrm{M}$ of vitamin $\mathrm{B}_{12}$ to the medium. Growth was observed by measuring the $\mathrm{OD}_{600}$ using the V-1200 spectrophotometer (VWR International, Darmstadt, Germany) by diluting the samples into an $\mathrm{OD}_{600}$ range of $0.05-0.25$. Additionally, $1-\mathrm{mL}$ samples were taken at the time points given in the results and centrifuged $(10 \mathrm{~min} ; 16.000 \times \mathrm{g})$, and the resulting supernatants were stored at $-20^{\circ} \mathrm{C}$ until further analysis.

\section{Recombinant DNA work}

All oligonucleotides used in this study were obtained from Eurofins MWG Operon (Ebersberg, Germany) or metabion international AG (Planegg, Germany) (Table 2). The plasmid construction was carried out with PCR fragments (KOD, Novagen, Darmstadt, Germany) generated with genomic DNA of C. glutamicum WT, E. coli DH5 $\alpha$ (DNA preparation described by [72]), or K. oxytoca DSM4798 (DSMZ, Braunschweig, Germany) as template DNA. These

Table 1 Strains and plasmids used in this study

\begin{tabular}{|c|c|c|}
\hline Strain or plasmid & Relevant characteristics & Source or reference \\
\hline \multicolumn{3}{|l|}{ C. glutamicum strains } \\
\hline WT & Wild type (ATCC13032) & [76] \\
\hline$\Delta \mathrm{cg} 1497$ & In-frame deletion of cg1497 in C. glutamicum WT & This work \\
\hline$\Delta h d p A$ & In-frame deletion of hdpA (cg2474) in C. glutamicum WT & This work \\
\hline$\Delta \mathrm{cg} 1497 \Delta h d p A$ & In-frame deletion of hdpA (cg2474) in C. glutamicum $\Delta \mathrm{cg} 1497$ & This work \\
\hline$\Delta h d p A \Delta / d h$ & In-frame deletion of Idh (cg3219) in C. glutamicum $\Delta h d p A$ & This work \\
\hline \multicolumn{3}{|l|}{ Plasmids } \\
\hline pK19mobsacB & $\begin{array}{l}\text { Kan }{ }^{a} \text {, mobilizable E. coli vector for the construction of insertion and deletion mutants of } \\
\text { C. glutamicum (oriV, sacB, lacZ) }\end{array}$ & [75] \\
\hline pEKEX3 & Spec $^{a} ;$ C. glutamicum/E. coli shuttle vector ( $P_{\text {tac }}$ lacl $^{b} ; \mathrm{pBL} 1$, OriV C.g.l $_{\text {OriV }}$ E.c. $)$ & [45] \\
\hline pWWEx1 & $\begin{array}{l}\text { Kan }{ }^{a} ; \text { C. glutamicum/E. coli shuttle vector for regulated gene expression }\left(P_{t a c},|a c|^{b} \text {, }\right. \\
\left.\text { pHM1519, oriV }{ }_{C . g,} \text { oriV } V_{E . c .}\right)\end{array}$ & [77] \\
\hline pK19mobsacB- $\Delta$ cg1497 & Kana, pK19mobsacB with the deletion construct for cg1497 & This work \\
\hline pK19mobsacB- $\Delta h d p A$ & $K_{a n}{ }^{a}$, pK19mobsacB with the deletion construct for hdpA (cg2474) & This work \\
\hline pK19mobsacB- $\Delta / d h$ & Kan ${ }^{a}$, pK19mobsacB with the deletion construct for Idh (cg3219) & {$[28]$} \\
\hline pEKEx3-mgsA-gldA & $\begin{array}{l}\text { Derived from pEKEx3 for IPTG-inducible overexpression of } m g s A \text { and } g / d A \text { from E. coli with } \\
\text { artificial ribosome binding site in front of each gene }\end{array}$ & This work \\
\hline pEKEx3-mgsA-yqhD-gldA & $\begin{array}{l}\text { Derived from pEKEx3 for IPTG-inducible overexpression of } m g s A \text {, yqhD, and gldA from E. coli } \\
\text { with artificial ribosome binding site in front of each gene }\end{array}$ & This work \\
\hline pEKEx3-mgsA-yqhD-fucO-gldA & $\begin{array}{l}\text { Derived from pEKEX3 for IPTG-inducible overexpression of mgsA, yahD, fucO, and gldA from } \\
\text { E. coli with artificial ribosome binding site in front of each gene }\end{array}$ & This work \\
\hline pWWEx1-ppdABC & $\begin{array}{l}\text { Derived from pEKEx3 for IPTG-inducible overexpression of ppdABC from K. oxytoca DSM4798 } \\
\text { with artificial ribosome binding site in front of the gene cluster }\end{array}$ & This work \\
\hline
\end{tabular}


Table 2 Oligonucleotides used in this study

\begin{tabular}{|c|c|c|}
\hline Oligonucleotide name & Sequence $\left(5^{\prime} \rightarrow 3^{\prime}\right)$ & Purpose \\
\hline cg1497_upstrm_fw_pK19 & GACTCTAGAGGATCCCCTTAACGCGCCGGGCTC & pK19mobsacB- $\Delta \mathrm{cg} 1497$ \\
\hline cg1497_upstrm_rv & GGGTAGGTGATTTGAATTTGTGCTITCGGAACTGGACATAATCAGATAC & pK19mobsacB- $\Delta \mathrm{cg} 1497$ \\
\hline cg1497_dwnstrm_fw & ACAAATTCAAATCACCTACCCGGAATGGAGAATCTGGTAGAGATCGG & pK19mobsacB- $\Delta \mathrm{cg} 1497$ \\
\hline cg1497_dwnstrm_rv_pK19 & CGAGCTCGGTACCCGAACTCTGGATGAGATAGCTGAGGTT & pK19mobsacB- $\Delta \mathrm{cg} 1497$ \\
\hline Dcg1497_fw_v3 & CCACTGCCACGGAGCC & Verification of cg1497 deletion by PCR \\
\hline Dcg1497_rv_v3 & AACGAAGTGCCACTTCTTCCAC & Verification of cg1497 deletion by PCR \\
\hline nagD_upstrm_fw_pK19 & GACTCTAGAGGATCCCCTTCCCCGCAATGAGCCG & pK19mobsacB- $\Delta h d p A$ \\
\hline nagD_upstrm_rv & GGGTAGGTGATTTGAATTTGTTGAAATGTTCACTGTCATAACACCATTGT & pK19mobsacB- $\Delta h d p A$ \\
\hline nagD_dwnstrm_fw & ACAAATTCAAATCACCTACCCTITCACGTACCAGATGAGCAGC & pK19mobsacB- $\Delta h d p A$ \\
\hline nagD_dwnstrm_rv_pK19 & CGAGCTCGGTACCCGGAACCTTCGGCTTGGATCTG & pK19mobsacB- $\Delta h d p A$ \\
\hline DnagD_fw & GATGAACACGACCGTTGCC & Verification of hdpA deletion by PCR \\
\hline DnagD_rv & GGGTGGTCTTTGAGGAGTTCTTC & Verification of hdpA deletion by PCR \\
\hline Idhfow & TGATGGCACCAGTTGCGATGT & Verification of Idh deletion by PCR \\
\hline Idhrev & CCATGATGCAGGATGGAGTA & Verification of Idh deletion by PCR \\
\hline mgsA_fw_x3 & $\begin{array}{l}\text { GACTCTAGAGGATCCCCGAAAGGAGGCCCTTCAGATGGAACTGACGA } \\
\text { CTCGCACT }\end{array}$ & $\begin{array}{l}\text { pEKEx3-mgsA-gldA, pEKEx3-mgsA-yqhD-gldA, } \\
\text { pEKEx3-mgsA-yqhD-fucO-gldA }\end{array}$ \\
\hline mgsA_rv_gld_DS & TATCTCATAAAGTTACTTCAGACGGTCCGCGA & pEKEx3-mgsA-gldA \\
\hline gldA_fw_mgs_DS & $\begin{array}{l}\text { GGACCGTCTGAAGTAACTTTATGAGATAGAAAGGAGGCCCTTCAGATGGAC } \\
\text { CGCATTATTCAATCACCG }\end{array}$ & pEKEx3-mgsA-gldA \\
\hline gldA_rv_x3 & CGAGCTCGGTACCCTTATTCCCACTCTTGCAGGAAAC & $\begin{array}{l}\text { pEKEx3-mgsA-gldA, pEKEx3-mgsA-yqhD-gldA, } \\
\text { pEKEx3-mgsA-yqhD-fucO-gldA }\end{array}$ \\
\hline mgsA_rv & TTACTTCAGACGGTCCGCGA & pEKEx3-mgsA-yqhD-gldA \\
\hline yqhD_fw_mgs & $\begin{array}{l}\text { GGACCGTCTGAAGTAAGAAAGGAGGCCCTTCAGATGAACAACTTTAATCTG } \\
\text { CACACCCC }\end{array}$ & pEKEx3-mgsA-yqhD-gldA \\
\hline yahD_rv & TTAGCGGGCGGCTTCGTATATA & pEKEx3-mgsA-yqhD-gldA \\
\hline gldA_fw_yqh & GCCGCCCGCTAAGAAAGGAGGCCCTTCAGATGGACCGCATTATTCAATCACCG & pEKEx3-mgsA-yqhD-gldA \\
\hline mgsA_rv_yqh_DS & TATCTCATAAAGTTACTTCAGACGGTCCGCGA & pEKEx3-mgsA-yqhD-fucO-gldA \\
\hline yqhD_fw_mgs_DS & $\begin{array}{l}\text { GGACCGTCTGAAGTAACTTTATGAGATAGAAAGGAGGCCCTTCAGATGAACAA } \\
\text { CTITAATCTGCACACCCC }\end{array}$ & pEKEx3-mgsA-yqhD-fucO-gldA \\
\hline yqhD_rv_gld_DS & GAAATGAATAGCTTAGCGGGCGGCTTCGTATATA & pEKEx3-mgsA-yqhD-fucO-gldA \\
\hline fucO_fw_ygh_DS & $\begin{array}{l}\text { GCCGCCCGCTAAGCTATTCATTTCGAAAGGAGGCCCTTCAGATGATGGCTAA } \\
\text { CAGAATGATTCTGAACG }\end{array}$ & pEKEx3-mgsA-yqhD-fucO-gldA \\
\hline fucO_rv_gld_DS & AAGGCAAGAATCTTACCAGGCGGTATGGTAAAGCT & pEKEx3-mgsA-yahD-fucO-gldA \\
\hline gldA_fw_fuc_DS & $\begin{array}{l}\text { CATACCGCCTGGTAAGATTCTTGCCTTGAAAGGAGGCCCTTCAGATGGACCGC } \\
\text { ATTATTCAATCACCG }\end{array}$ & pEKEx3-mgsA-yqhD-fucO-gldA \\
\hline ppdABC_ko_fw_x1 & $\begin{array}{l}\text { CTGCAGGTCGACTCTAGAGGAAAGGAGGCCCTTCAGATGAGATCGAAAAG } \\
\text { ATTGAAGCACTGG }\end{array}$ & pWWEx1-ppdABC \\
\hline ppdABC_ko_rv_x1 & CGGTACCCGGGGATCTTAATCGTCGCCTTTGAGTTTTTIACG & pWWEx1-ppdABC \\
\hline gldA_Seq & GAACTGTGCTACAACACCCTG & Sequencing primer for gldA \\
\hline yqhD_Seq & GTATTTGCCGTGCTCGATC & Sequencing primer for yqhD \\
\hline fucO_Seq & GACCAATAAACCCAGTGTAC & Sequencing primer for fucO \\
\hline ppdABC_Seq1 & CGAACAGGAAACCACCGTTG & Sequencing primer for $p p d A B C$ \\
\hline ppdABC_Seq2 & ACGACCAGACCTTCACCCAC & Sequencing primer for $p p d A B C$ \\
\hline ppdABC_Seq3 & TACCTGCATACCTCCGCGAT & Sequencing primer for $p p d A B C$ \\
\hline ppdABC_Seq4 & AATCCTCCGACGTGGCCTTC & Sequencing primer for $p p d A B C$ \\
\hline ppdABC_Seq5 & CGAACAAGCACCCGGAATGG & Sequencing primer for $p p d A B C$ \\
\hline
\end{tabular}


Table 2 Oligonucleotides used in this study (Continued)

\begin{tabular}{|c|c|c|}
\hline pWWEx1_fw & CATCATAACGGTTCTGGC & $\begin{array}{l}\text { Verification of correct pEKEx3/pWWEx } 1 \\
\text { derivatives by PCR/sequencing }\end{array}$ \\
\hline pWWEx1_rv & ATCTTCTCTCATCCGCCA & $\begin{array}{l}\text { Verification of correct pEKEx } 3 / p \text { WEXX1 } \\
\text { derivatives by PCR/sequencing }\end{array}$ \\
\hline M13_fw & CGCCAGGGTITCCCAGTCACGAC & $\begin{array}{l}\text { Verification of correct pK19mobsacB } \\
\text { derivatives by PCR/sequencing }\end{array}$ \\
\hline M13_rv & AGCGGATAACAATTTCACACAGGA & $\begin{array}{l}\text { Verification of correct pK19mobsacB } \\
\text { derivatives by PCR/sequencing }\end{array}$ \\
\hline
\end{tabular}

Sequence in italics: overlapping sequences for Gibson-Assembly; sequence bold italics: artificial ribosome binding site

fragments were cloned via Gibson Assembly [73] (enzymes provided by NEB, Frankfurt am Main, Germany) into the linearized vectors, and the resulting reaction was used for the transformation of $E$. coli DH5 $\alpha$ cells using the calcium chloride method [70]. Therefore, pEKEx3 and pK19mob$s a c B$ were digested with the restriction enzyme SmaI and pVWEx1 with BamHI (Fermentas/Thermo Scientific, St. Leon-Rot, Germany). For the purification of the PCR fragments and the digested plasmids, the PCR purification kit or MinElute PCR purification kit (QIAGEN, Hilden, Germany) were applied. The plasmids were isolated from E. coli by using the QIAprep spin miniprep kit (QIAGEN, Hilden, Germany). All resulting vectors were sequenced to confirm the correctness of the cloned DNA fragments (SCF, CeBiTec, Bielefeld, Germany). The transformation of C. glutamicum was performed with electrocompetent cells [74] by electroporation [71] in a GenePulser Xcell ${ }^{\mathrm{mm}}$ plus PC Module (BioRad, München, Germany) but using LB with $2 \%$ glucose in all stages of cultivation. All enzymes and kit systems were used like recommended in the manufacturer's manuals.

\section{Construction of $C$. glutamicum deletion strains}

To delete the genes cg1497 and $h d p A$ new plasmids were constructed by using the suicide vector pK19mobsacB [75]. For the deletion of cg1497, genomic regions flanking this gene were amplified via PCR from genomic DNA of C. glutamicum using the primer pairs cg1497_upstrm fw_pK19/cg1497_upstrm_rv and cg1497_dwnstrm_fw/ cg1497_dwnstrm_rv_pK19 (Table 2). The resulting PCR fragments were purified and cloned via Gibson-Assembly into the linearized vector $\mathrm{pK} 19 \mathrm{mobsacB}$ resulting in the plasmid pK19mobsacB- $\Delta \operatorname{cg} 1497$ (Table 1). The deletion of the gene cg1497 was carried out with this plasmid by a two-step homologous recombination procedure described before [71]. For the verification of the correct in-frame deletion of the gene cg1497, a PCR (Taq DNA polymerase with ThermoPol ${ }^{\circ}$ Buffer, NEB, Frankfurt am Main, Germany) was performed using the primer pair Dcg1497_fw_v3/Dcg1497_rv_v3 (Table 2). Accordingly the deletion of $h d p A$ (cg2474) was realized, using the primer pairs nagD_upstrm_fw_pK19/nagD_upstrm_rv and nagD_dwnstrm_fw/nagD_dwnstrm_rv_pK19 (Table 2) for the cloning procedure of the plasmid pK19mobsacB$\Delta h d p A$ (Table 1) and the primer pair DnagD_fw/DnagD_rv (Table 2) for the verification of the in-frame deletion via PCR. The plasmid pK19mobsacB- $\Delta l d h$ (Table 1) was already available [28]. Thus, the primer pair ldhfow/ldhrev (Table 2) was used to verify the successful in-frame deletion of $l d h$ after the two-step homologous recombination.

\section{GC-MS measurements}

The supernatants of the samples taken in the cultivation were analyzed using a TRACE GC ULTRA connected to an AS 3000 Auto-sampler and to an ISQ Single Quadrupole Mass Spectrometer using a TG-WAXMS (length: $30 \mathrm{~m}$; I.D.: $0.25 \mathrm{~mm}$; film: $0.25 \mu \mathrm{m}$ ) (Thermo Scientific, Dreieich, Germany). The thawed supernatants were directly diluted 1:10 with methanol (HPLC gradient grade; VWR Chemicals, Fontenay-sous-Bois, France) or after an additional 1:10 dilution step with water (Milli-Q grade). Prior to injection, the diluted samples were centrifuged $(10 \mathrm{~min} ; 16,000 \times \mathrm{g})$ and the resulting supernatant was used for analysis. The operating setup was the following: the temperature of the MS transfer line and the ion source were hold at $230{ }^{\circ} \mathrm{C}$; the injector temperature was set to $220{ }^{\circ} \mathrm{C}$ and a gradient was used for the oven (holding $40{ }^{\circ} \mathrm{C}$ for $1 \mathrm{~min}$, increasing the temperature with a rate of $12{ }^{\circ} \mathrm{C} / \mathrm{min}$ up to $230{ }^{\circ} \mathrm{C}$ and holding this for $5 \mathrm{~min}$ ); in the constant flow mode, the flow rate of the carrier gas helium was $1 \mathrm{~mL} / \mathrm{min}$ using the splitless mode of the injector (split flow: $10 \mathrm{~mL} / \mathrm{min}$; splitless time: $1.5 \mathrm{~min}$; focus liner: $5 \times 8 \times 105 \mathrm{~mm}$, splitless for $50-\mathrm{mm}$ needle with glass wool); the electron impact ionization energy was $70 \mathrm{eV}$. The compounds 1,2-propanediol and acetol were measured with this method by creating a calibration curve with an external standard. The peaks were identified by retention time and were quantified using the intensity of one specific $\mathrm{m} / \mathrm{z}$ value (1,2-propanediol: $\mathrm{m} / \mathrm{z}=45$; acetol: $\mathrm{m} / \mathrm{z}=43)$. For the computational quantification, the program Xcalibur 2.1 (2.1.0 SP1.1160, Thermo Scientific, Dreieich, Germany) was employed.

\section{HPLC measurements}

The compounds glucose, glycerol, DHA, lactate, propanal, and 1-propanol were quantified with a HPLC system 
(1200 series, Agilent Technologies, Böblingen, Germany). As a immobile phase, an organic acid resin column $(300 \times 8 \mathrm{~mm})$ with the appropriate pre-column $(40 \times$ $8 \mathrm{~mm}$ ) (Chromatographie-Service $\mathrm{GmbH}$, Langerwehe, Germany) was installed and heated up to $60{ }^{\circ} \mathrm{C}$ while the mobile phase was $5 \mathrm{mM}$ sulfuric acid in water (Milli-Q grade) with a flow rate of $0.8 \mathrm{~mL} / \mathrm{min}$ or $1 \mathrm{~mL} / \mathrm{min}$. The signals were acquired with a refractive index detector (glucose, glycerol, propanal, and 1-propanol) and a diode array detector at a signal wavelength of $210 \mathrm{~nm}$ and a reference wavelength of $360 \mathrm{~nm}$ (DHA, lactate). For the calibration curve, external standards for every compound were prepared and the supernatants of the samples from the cultivations were measured undiluted after thawing.

\begin{abstract}
Abbreviations
$\Delta$ : deletion; ADP: adenosine diphosphate; ATP: adenosine triphosphate; butA: gene coding for (S,S)-butanediol dehydrogenase (ButA); CeBiTec: Center for Biotechnology; cg1497: gene coding for predicted kinase related to dihydroxyacetone kinase; C. glutamicum: Conynebacterium glutamicum; CoA: Coenzyme A; cgR_2242: gene coding for putative aldo-keto reductase (AKR); DHA(P): dihydroxyacetone (phosphate); DNA: deoxyribonucleic acid; DSMZ: German Collection of Microorganisms and Cell Cultures; E. coll: Escherichia coli; fucO: gene coding for propanediol oxidoreductase/lactaldehyde reductase (FuCO); GC-MS: gas chromatography-mass spectrometry; gldA: gene coding for glycerol dehydrogenase (GldA); gpp: gene coding for glycerol-3-phosphatase (Gpp); hdpA: Gene coding for dihydroxyacetone phosphate phosphatase (HdpA); HPLC: High-performance liquid chromatography; IPTG: isopropyl- $\beta$-Dthiogalactopyranoside; K. oxytoca: Klebsiella oxytoca; LB: lysogeny broth complex medium; ldh: gene coding for L-lactate dehydrogenase (LdhA); mgsA: gene coding for methylglyoxal synthase (MgsA); mshA: gene encoding mycothiol glycosyltransferase (MshA); NADH and NAD: reduced or oxidized form of nicotinamide adenine dinucleotide, respectively; NADPH and NADP: reduced and oxidized form of nicotinamide adenine dinucleotide phosphate, respectively; NEB: New England Biolabs; $\mathrm{OD}_{600}$ : optical density at wavelength $(\lambda) 600 \mathrm{~nm}$; PCR: polymerase chain reaction; PntAB: transmembrane transhydrogenase; ppdABC: operon coding for diol dehydratase (PpdABC); PPP: pentose phosphate pathway; Pyk: pyruvate kinase; rpm: revolutions per minute; SCF: Sequencing Core Facility; TCA: citric acid cycle; Vit. $B_{12}$ : vitamin $B_{12}$ WT: wild type; yqhD: gene coding for aldehyde reductase (YqhD).
\end{abstract}

\section{Competing interests}

The authors declare that they have no competing interests.

\section{Authors' contributions}

DS carried out all experimental work and data analysis, as well as drafted and revised the manuscript. VFW conceived, supervised, and managed the study, as well as revised and finalized the manuscript. Both authors read and approved the manuscript.

\section{Acknowledgements}

This study was funded by the German Federal Ministry of Education and Research (BMBF, Grant. No. 0316017). The authors thank Dr. Steffen Lindner for the initiation of this study. We acknowledge support of the publication fee by Deutsche Forschungsgemeinschaft and the Open Access Publication Funds of Bielefeld University.

Received: 20 April 2015 Accepted: 5 June 2015

Published online: 24 June 2015

\section{References}

1. Soucaille P, Meynial SI, Voelker F, Figge R, inventors. Microorganisms and methods for production of 1,2-propanediol and acetol. 2008.

2. Saxena RK, Anand P, Saran S, Isar J, Agarwal L. Microbial production and applications of 1,2-propanediol. Indian J Microbiol. 2010;50:2-11. doi:10.1007/s12088-010-0017-x.
3. Lloyd L. Handbook of industrial catalysts. Boston: Springer Science + Business Media LLC; 2011.

4. Chauvel A, Lefebvre G. Petrochemical processes: volume 2: major oxygenated, chlorinated and nitrated derivatives. Paris: Éditions Technip; 1989.

5. Bennett GN, San KY. Microbial formation, biotechnological production and applications of 1,2-propanediol. Appl Microbiol Biotechnol. 2001;55:1-9.

6. Behr A, Eilting J, Irawadi K, Leschinski J, Lindner F. Improved utilisation of renewable resources: new important derivatives of glycerol. Green Chem. 2008;10:13-30. doi:10.1039/B710561D.

7. Cameron DC, Cooney CL. A novel fermentation: the production of $\mathrm{R}(-)-1,2-$ propanediol and acetol by Clostridium thermosaccharolyticum. Nat Biotechnol. 1986;4:651-4. doi:10.1038/nbt0786-651.

8. Jung J, Choi E, Oh M. Enhanced production of 1,2-propanediol by tpi1 deletion in Saccharomyces cerevisiae. J Microbiol Biotechnol. 2008;18:1797-802.

9. Jung J, Yun HS, Lee J, Oh M. Production of 1,2-propanediol from glycerol in Saccharomyces cerevisiae. J Microbiol Biotechnol. 2011;21:846-53.

10. Clomburg JM, Gonzalez R. Metabolic engineering of Escherichia coli for the production of 1,2-propanediol from glycerol. Biotechnol Bioeng. 2011;108:867-79. doi:10.1002/bit.22993.

11. Li H, Liao JC. Engineering a cyanobacterium as the catalyst for the photosynthetic conversion of $\mathrm{CO}_{2}$ to 1,2-propanediol. Microb Cell Fact. 2013;12:4. doi:10.1186/1475-2859-12-4

12. Niimi S, Suzuki N, Inui M, Yukawa H. Metabolic engineering of 1,2-propanediol pathways in Conynebacterium glutamicum. Appl Microbiol Biotechnol. 2011;90:1721-9. doi:10.1007/s00253-011-3190-x.

13. Kinoshita S, Udaka S, Shimono M. Studies on the amino acid fermentation. J Gen Appl Microbiol. 1957;3:193-205. doi:10.2323/jgam.3.193.

14. Wendisch VF. Microbial production of amino acids and derived chemicals: synthetic biology approaches to strain development. Curr Opin Biotechnol. 2014;30C:51-8. doi:10.1016/j.copbio.2014.05.004.

15. Eggeling L, Bott M, editors. Handbook of Corynebacterium glutamicum. Boca Raton, Fla: Taylor \& Franics; 2005

16. Mitsuhashi S. Current topics in the biotechnological production of essentia amino acids, functional amino acids, and dipeptides. Curr Opin Biotechnol. 2014;26:38-44. doi:10.1016/j.copbio.2013.08.020.

17. Becker J, Wittmann C. Biotechnologie von Morgen: metabolisch optimierte Zellen für die bio-basierte Produktion von Chemikalien und Treibstoffen, Materialien und Gesundheitsprodukten. Angew Chem. 2015;127:3383-407. doi:10.1002/ange.201409033.

18. Burkovski A. Corynebacterium glutamicum: From Systems Biology to Biotechnological Applications. Portland: Caister Academic Press; 2015.

19. Kalinowski J, Bathe B, Bartels D, Bischoff N, Bott M, Burkovski A, et al. The complete Corynebacterium glutamicum ATCC 13032 genome sequence and its impact on the production of L-aspartate-derived amino acids and vitamins. J Biotechnol. 2003;104:5-25. doi:10.1016/S0168-1656(03)00154-8.

20. Unthan S, Baumgart M, Radek A, Herbst M, Siebert D, Brühl N, et al. Chassis organism from Conynebacterium glutamicum - a top-down approach to identify and delete irrelevant gene clusters. Biotechnol J. 2014. doi:10.1002/biot.201400041.

21. Becker J, Wittmann C. Systems and synthetic metabolic engineering for amino acid production - the heartbeat of industrial strain development. Curr Opin Biotechnol. 2012;23:718-26. doi:10.1016/j.copbio.2011.12.025.

22. Mimitsuka T, Sawai H, Hatsu M, Yamada K. Metabolic engineering of Corynebacterium glutamicum for cadaverine fermentation. Biosci Biotechnol Biochem. 2007;71:2130-5. doi:10.1271/bbb.60699.

23. Schneider J, Wendisch VF. Putrescine production by engineered Corynebacterium glutamicum. Appl Microbiol Biotechnol. 2010;88:859-68. doi:10.1007/s00253-010-2778-x.

24. Wieschalka S, Blombach B, Bott M, Eikmanns BJ. Bio-based production of organic acids with Corynebacterium glutamicum. Microb Biotechnol. 2013;6:87-102. doi:10.1111/1751-7915.12013.

25. Heider SA, Wolf N, Hofemeier A, Peters-Wendisch P, Wendisch VF. Optimization of the IPP precursor supply for the production of lycopene, decaprenoxanthin and astaxanthin by Conynebacterium glutamicum. Front Bioeng Biotechnol. 2014;2:28. doi:10.3389/fbioe.2014.00028.

26. Smith KM, Cho K, Liao JC. Engineering Corynebacterium glutamicum for isobutanol production. Appl Microbiol Biotechnol. 2010;87:1045-55. doi:10.1007/s00253-010-2522-6.

27. Yamamoto S, Suda M, Niimi S, Inui M, Yukawa H. Strain optimization for efficient isobutanol production using Conynebacterium glutamicum under oxygen deprivation. Biotechnol Bioeng. 2013;110:2938-48. doi:10.1002/bit.24961. 
28. Blombach B, Riester T, Wieschalka S, Ziert C, Youn J, Wendisch VF, et al. Corynebacterium glutamicum tailored for efficient isobutanol production. Appl Environ Microbiol. 2011;77:3300-10. doi:10.1128/AEM.02972-10.

29. Inui M, Kawaguchi $H$, Murakami S, Vertès AA, Yukawa H. Metabolic engineering of Corynebacterium glutamicum for fuel ethanol production under oxygen-deprivation conditions. J Mol Microbiol Biotechnol. 2004;8:243-54. doi:10.1159/000086705.

30. Sakai S, Tsuchida Y, Nakamoto H, Okino S, Ichihashi O, Kawaguchi H, et al. Effect of lignocellulose-derived inhibitors on growth of and ethanol production by growth-arrested Conyebacterium glutamicum R. Appl Environ Microbiol. 2007;73:2349-53. doi:10.1128/AEM.02880-06.

31. Jojima T, Noburyu R, Sasaki M, Tajima T, Suda M, Yukawa H, et al. Metabolic engineering for improved production of ethanol by Corynebacterium glutamicum. Appl Microbiol Biotechnol. 2015;99:1165-72. doi:10.1007/ s00253-014-6223-4

32. Zahoor A, Lindner SN, Wendisch VF. Metabolic engineering of Corynebacterium glutamicum aimed at alternative carbon sources and new products. Comput Struct Biotechnol J. 2012;3:e201210004. doi:10.5936/ csbj.201210004

33. Jain R, Yan Y. Dehydratase mediated 1-propanol production in metabolically engineered Escherichia coli. Microb Cell Fact. 2011;10:97. doi:10.1186/14752859-10-97.

34. Ammar EM, Wang Z, Yang S. Metabolic engineering of Propionibacterium freudenreichii for n-propanol production. Appl Microbiol Biotechnol. 2013;97:4677-90. doi:10.1007/s00253-013-4861-6.

35. Srirangan K, Liu X, Westbrook A, Akawi L, Pyne ME, Moo-Young M, et al. Biochemical, genetic, and metabolic engineering strategies to enhance coproduction of 1-propanol and ethanol in engineered Escherichia coli. Appl Microbiol Biotechnol. 2014;98:9499-515. doi:10.1007/s00253-0146093-9.

36. Shen CR, Liao JC. Synergy as design principle for metabolic engineering of 1-propanol production in Escherichia coli. Metab Eng. 2013;17:12-22. doi:10.1016/j.ymben.2013.01.008.

37. Choi YJ, Park JH, Kim TY, Lee SY. Metabolic engineering of Escherichia coli for the production of 1-propanol. Metab Eng. 2012;14:477-86. doi:10.1016/ j.ymben.2012.07.006.

38. Lindner SN, Meiswinkel TM, Panhorst M, Youn J, Wiefel L, Wendisch VF. Glycerol-3-phosphatase of Corynebacterium glutamicum. J Biotechnol. 2012;159:216-24. doi:10.1016/j.jbiotec.2012.02.003.

39. Jojima T, Igari T, Moteki Y, Suda M, Yukawa H, Inui M. Promiscuous activity of $(S, S)$-butanediol dehydrogenase is responsible for glycerol production from 1,3-dihydroxyacetone in Corynebacterium glutamicum under oxygendeprived conditions. Appl. Microbiol. Biotechnol. 2014. doi:10.1007/s00253014-6170-0.

40. Subedi KP, Kim I, Kim J, Min B, Park C. Role of GldA in dihydroxyacetone and methylglyoxal metabolism of Escherichia coli K12. FEMS Microbiol Lett. 2008;279:180-7. doi:10.1111/j.1574-6968.2007.01032.x.

41. Thomas Dr. Haas, Li Dr. Li, Achim Dr. Marx, Juraj Obuch, Volker F. Prof. Dr. Wendisch, inventors. Preparing dihydroxyacetone, useful e.g. in cosmetic composition, comprises cultivating microorganisms in growth medium, adjusting $\mathrm{pH}$ of the medium, contacting cells with a base and culturing the microorganism in presence of carbohydrates. 2008

42. Jojima T, Igari T, Gunji W, Suda M, Inui M, Yukawa $H$. Identification of a HAD superfamily phosphatase, HdpA, involved in 1,3-dihydroxyacetone production during sugar catabolism in Corynebacterium glutamicum. FEBS Lett. 2012;586:4228-32. doi:10.1016/j.febslet.2012.10.028.

43. Pauling J, Röttger R, Tauch A, Azevedo V, Baumbach J. CoryneRegNet 6.0-Updated database content, new analysis methods and novel features focusing on community demands. Nucleic Acids Res. 2012;40:D610-4. doi:10.1093/nar/gkr883.

44. Okino S, Inui M, Yukawa H. Production of organic acids by Corynebacterium glutamicum under oxygen deprivation. Appl Microbiol Biotechnol. 2005;68:475-80. doi:10.1007/s00253-005-1900-y.

45. Stansen C, Uy D, Delaunay S, Eggeling L, Goergen J, Wendisch VF. Characterization of a Corynebacterium glutamicum lactate utilization operon induced during temperature-triggered glutamate production. Appl Environ Microbiol. 2005;71:5920-8. doi:10.1128/AEM.71.10.5920-5928.2005.

46. Kato O, Youn J, Stansen KC, Matsui D, Oikawa T, Wendisch VF. Quinonedependent D-lactate dehydrogenase Dld (Cg1027) is essential for growth of Corynebacterium glutamicum on D-lactate. BMC Microbiol. 2010;10:321. doi:10.1186/1471-2180-10-321.
47. Jain R, Sun X, Yuan Q, Yan Y. Systematically engineering Escherichia coli for enhanced production of 1,2-propanediol and 1-propanol. ACS Synth Biol. 2014. doi:10.1021/sb500345t.

48. Jarboe LR. YqhD: a broad-substrate range aldehyde reductase with various applications in production of biorenewable fuels and chemicals. Appl Microbiol Biotechnol. 2011;89:249-57. doi:10.1007/s00253-010-2912-9.

49. Tokuyama K, Ohno S, Yoshikawa K, Hirasawa T, Tanaka S, Furusawa C, et al. Increased 3-hydroxypropionic acid production from glycerol, by modification of central metabolism in Escherichia coli. Microb Cell Fact. 2014;13:64. doi:10.1186/1475-2859-13-64

50. Feng X, Xian M, Liu W, Xu C, Zhang H, Zhao G. Biosynthesis of poly(3hydroxypropionate) from glycerol using engineered Klebsiella pneumoniae strain without vitamin $B_{12}$. Bioengineered. 2015. doi:10.1080/ 21655979.2015.1011027.

51. Foo JL, Jensen HM, Dahl RH, George K, Keasling JD, Lee TS, et al. Improving microbial biogasoline production in Escherichia coli using tolerance engineering. MBio. 2014;5:e01932. doi:10.1128/mBio.01932-14.

52. Zhu H, Yi X, Liu Y, Hu H, Wood TK, Zhang X. Production of acetol from glycerol using engineered Escherichia coli. Bioresour Technol. 2013;149:238-43. doi:10.1016/j.biortech.2013.09.062.

53. Okino S, Noburyu R, Suda M, Jojima T, Inui M, Yukawa H. An efficient succinic acid production process in a metabolically engineered Corynebacterium glutamicum strain. Appl Microbiol Biotechnol. 2008;81:459-64. doi:10.1007/s00253-008-1668-y.

54. Okino S, Suda M, Fujikura K, Inui M, Yukawa H. Production of D-lactic acid by Corynebacterium glutamicum under oxygen deprivation. Appl Microbiol Biotechnol. 2008;78:449-54. doi:10.1007/s00253-007-1336-7.

55. Netzer R, Krause M, Rittmann D, Peters-Wendisch PG, Eggeling L, Wendisch VF, et al. Roles of pyruvate kinase and malic enzyme in Corynebacterium glutamicum for growth on carbon sources requiring gluconeogenesis. Arch Microbiol. 2004;182:354-63. doi:10.1007/s00203-004-0710-4.

56. Gubler M, Jetten M, Lee SH, Sinskey AJ. Cloning of the pyruvate kinase gene (pyk) of Corynebacterium glutamicum and site-specific inactivation of pyk in a lysine-producing Corynebacterium lactofermentum strain. Appl Environ Microbiol. 1994;60:2494-500.

57. Grabar TB, Zhou S, Shanmugam KT, Yomano LP, Ingram LO. Methylglyoxal bypass identified as source of chiral contamination in $L(+)$ and $D(-)$-lactate fermentations by recombinant Escherichia coli. Biotechnol Lett. 2006;28:1527-35. doi:10.1007/s10529-006-9122-7.

58. Vetting MW, Frantom PA, Blanchard JS. Structural and enzymatic analysis of MshA from Corynebacterium glutamicum: substrate-assisted catalysis. J Biol Chem. 2008;283:15834-44. doi:10.1074/jbc.M801017200.

59. Lessmeier L, Hoefener M, Wendisch VF. Formaldehyde degradation in Corynebacterium glutamicum involves acetaldehyde dehydrogenase and mycothiol-dependent formaldehyde dehydrogenase. Microbiology. 2013;159:2651-62. doi:10.1099/mic.0.072413-0.

60. Witthoff S, Mühlroth A, Marienhagen J, Bott M. C1 metabolism in Corynebacterium glutamicum: an endogenous pathway for oxidation of methanol to carbon dioxide. Appl Environ Microbiol. 2013;79:6974-83. doi:10.1128/AEM.02705-13.

61. Liu Y, Chen C, Chaudhry MT, Si M, Zhang L, Wang Y, et al. Enhancing Corynebacterium glutamicum robustness by over-expressing a gene, mshA, for mycothiol glycosyltransferase. Biotechnol Lett. 2014;36:1453-9. doi:10.1007/s10529-014-1501-X.

62. Shibata N, Masuda J, Morimoto Y, Yasuoka N, Toraya T. Substrate-induced conformational change of a coenzyme B 12 -dependent enzyme: crystal structure of the substrate-free form of diol dehydratase. Biochemistry. 2002;41:12607-17. doi:10.1021/bi026104z.

63. Tobimatsu T, Sakai T, Hashida Y, Mizoguchi N, Miyoshi S, Toraya T. Heterologous expression, purification, and properties of diol dehydratase, an adenosylcobalamin-dependent enzyme of Klebsiella oxytoca. Arch Biochem Biophys. 1997;347:132-40. doi:10.1006/abbi.1997.0325.

64. Shibata N, Nakanishi Y, Fukuoka M, Yamanishi M, Yasuoka N, Toraya T. Structural rationalization for the lack of stereospecificity in coenzyme $B_{12}$-dependent diol dehydratase. J Biol Chem. 2003;278:22717-25. doi:10.1074/jbc.M301513200.

65. Kabus A, Georgi T, Wendisch VF, Bott M. Expression of the Escherichia coli pnt $A B$ genes encoding a membrane-bound transhydrogenase in Corynebacterium glutamicum improves L-lysine formation. Appl Microbiol Biotechnol. 2007;75:47-53. doi:10.1007/s00253-006-0804-9.

66. Marx A, Hans S, Möckel B, Bathe B, De G, Albert A. Metabolic phenotype of phosphoglucose isomerase mutants of Corynebacterium glutamicum. J Biotechnol. 2003;104:185-97. doi:10.1016/S0168-1656(03)00153-6. 
67. Bommareddy RR, Chen Z, Rappert S, Zeng A. A de novo NADPH generation pathway for improving lysine production of Corynebacterium glutamicum by rational design of the coenzyme specificity of glyceraldehyde 3-phosphate dehydrogenase. Metab Eng. 2014;25:30-7. doi:10.1016/j.ymben.2014.06.005.

68. Shi F, Huan X, Wang X, Ning J. Overexpression of NAD kinases improves the L-isoleucine biosynthesis in Corynebacterium glutamicum ssp. lactofermentum. Enzyme Microb Technol. 2012;51:73-80. doi:10.1016/j.enzmictec.2012.04.003.

69. Hanahan D. Studies on transformation of Escherichia coli with plasmids. J Mol Biol. 1983;166:557-80. doi:10.1016/S0022-2836(83)80284-8.

70. Sambrook J, Russell DW. Molecular cloning: a laboratory manual. 3rd ed. Cold Spring Harbor: Cold Spring Harbor Laboratory Press; 2001.

71. Eggeling L, Reyes O. Experiments. In: Eggeling L, Bott M, editors. Handbook of Corynebacterium glutamicum. Boca Raton, Fla: Taylor \& Franics; 2005. p. 535-68.

72. Eikmanns BJ, Thum-Schmitz N, Eggeling L, Ludtke K, Sahm H. Nucleotide sequence, expression and transcriptional analysis of the Corynebacterium glutamicum gltA gene encoding citrate synthase. Microbiology. 1994;140:1817-28. doi:10.1099/13500872-140-8-1817.

73. Gibson DG, Young L, Chuang R, Venter JC, Hutchison CA, Smith HO. Enzymatic assembly of DNA molecules up to several hundred kilobases. Nat Methods. 2009;6:343-5. doi:10.1038/NMETH.1318.

74. Follettie MT, Peoples OP, Agoropoulou C, Sinskey AJ. Gene structure and expression of the Corynebacterium flavum N13 ask-asd operon. J Bacteriol. 1993;175:4096-103.

75. Schäfer A, Tauch A, Jäger W, Kalinowski J, Thierbach G, Pühler A. Small mobilizable multi-purpose cloning vectors derived from the Escherichia coli plasmids pK18 and pK19: selection of defined deletions in the chromosome of Corynebacterium glutamicum. Gene. 1994;145:69-73. doi:10.1016/03781119(94)90324-7.

76. Abe S, Takayama K, Kinoshita S. Taxanomical studies on glutamic acidproducing bacteria. J Gen Appl Microbiol. 1967;13:279-301. doi:10.2323/ jgam.13.279

77. Peters-Wendisch PG, Schiel B, Wendisch VF, Katsoulidis E, Möckel B, Sahm H, et al. Pyruvate carboxylase is a major bottleneck for glutamate and lysine production by Corynebacterium glutamicum. J Mol Microbiol Biotechnol. 2001;3:295-300

\section{Submit your next manuscript to BioMed Central and take full advantage of:}

- Convenient online submission

- Thorough peer review

- No space constraints or color figure charges

- Immediate publication on acceptance

- Inclusion in PubMed, CAS, Scopus and Google Scholar

- Research which is freely available for redistribution

Submit your manuscript at www.biomedcentral.com/submit 